\title{
QUALITATIVE RESEARCH IN TRAVEL BEHAVIOR STUDIES
}

\author{
María del Lidón Mars Aicart \\ Psychologist PhD, Universitat de València, Spain \\ María Rosa Arroyo López \\ Researcher, Universitat Politècnica de València, Spain \\ Tomás Ruiz Sánchez \\ Associate Professor, Universitat Politècnica de València, Spain
}

\begin{abstract}
Qualitative methodology is extensively used in a wide range of scientific areas, such as Sociology and Psychology, and it is been used to study individual and household decision making processes. However, in the Transportation Planning and Engineering domain it is still infrequent to find in the travel behavior literature studies using qualitative techniques to explore activity-travel decisions.
\end{abstract}

The aim of this paper is first, to provide an overview of the types of qualitative techniques available and to explore how to correctly implement them. Secondly, to highlight the special characteristics of qualitative methods that make them appropriate to study activity-travel decision processes. Far from been an unempirical or intuitive methodology, using qualitative methods properly implies a strong foundation on theoretical frameworks, a careful design of data collection and a deep data analysis. For such a purpose, a review of the scarce activitytravel behavior literature using qualitative methods, or a combination of qualitative and quantitative approaches, is presented.

The use of qualitative techniques can play a role of being a supplementary way of obtaining information related to activity-travel decisions which otherwise it would be extremely difficult to find. This work ends with some conclusions about how qualitative research could help in making progress on activity-travel behavior studies.

\section{INTRODUCTION}

Methods of analysis based on qualitative approaches to develop travel behavior studies are increasingly used. These methods are sufficiently open to address complex subjects, and they are especially suited to analyze interactions of individuals in everyday life considering concrete contexts (Flick, 2014). The subjectivity of the qualitative researchers, which is usually criticized, becomes part of the research process, increasing the richness of the data (Madill, Jordan \& Shirley, 2000; Madill, \& Gough, 2008).

Qualitative methods applied to travel behavior studies focus on the subjective experiences 
of individuals related to travel. On the other hand, quantitative approaches are more interested in knowing frequency and distributions of trips. The former methods are especially appropriate for answering micro questions and the latter for answering macro questions. Nevertheless, both approaches can be used either as separate techniques or as multidisciplinary parts of a wider study (Grosvenor, 2000). Qualitative methods could be used for explaining the relations that quantitative methods find. They can also be used prior to a questionnaire administration, to determine the best way of stating the questions. Qualitative methods can be useful for example to focus more deeply on some answers through open questions added to quantitative questionnaires. So it is common to use both methodologies either one after the other or at the same time. Therefore both methods can be used together although they remain autonomous (i.e. Grosvenor, 2000; Niglas, 2000; HesseBiber, 2010).

Qualitative approaches are broadly classified on descriptive and interpretative studies. The former nearly do not present research results including interpretation or conceptualization (i.e Ethnographies). The latter use research data to illustrate existing theories or concepts (Analytic Induction), or to derive those theories from the analysis of the data without any hypothesis (Grounded Theory) (i.e. Amezcua \& Galvez Toro, 2002)

Content Analysis (Berelson, 1952) consists on a set of methods based on studying words, text meaning or context, which can be used in both descriptive and interpretative studies. It is a technique that systematically and objectively identifies specified characteristics of the material. It may transform the information into categories allowing the conversion of the information into quantitative data such us frequencies or ratings (Smith, 2000). Grounded Theory (Glaser and Strauss, 1967) generates concepts and hypothesis using inductive analysis. No prior research results, hypothesis or existing theoretical frameworks are used. It is defined by a number of characteristics that allow researchers to make sense a huge amount of data, develop or test their ideas about data (Charmaz, 1996). Analytic Induction (AI) (Znaniecki, 1934) uses empirical data to check existing theories, and the data is used to expand and generalized the results found. Taylor and Bogdan's (1984) methodology is an example of AI.

Besides Content Analysis, other qualitative interpretation approaches include: Conversation Analysis that focuses on particular socio-linguistic phenomenon; Thematic Coding is a method for identifying, analyzing and reporting patterns (themes) within data; Analysis of Narrative Interviews, and Objective Hermeneutics, the focus is on conducting case studies; Discourse Analysis, data are analyzed at a macrosociological level, as social texts (Patton, 2005).

Using qualitative methods is not straightforward. It is necessary to justify its use in relation to the aim of the project. For a good practice and a better understanding of the reader it is essential define accurately all the stages that constitutes the qualitative process. Firstly the 
recruitment process of participants and the survey tools have to be described. In particular, it is important to mention any quality-related aspect of data collection. The data analysis process should be clear. Quality assurances in the research should be described and it is needed to summarize all findings and to draw practical consequences and discuss them. After that it is advisable to specify which qualitative method it is being used and how has it been carried out. As well as triangulation strategies used to verify data and avoid bias. Finally, it might provide a further understanding to itemize the coding process, the main themes, and the categories tree or the way they are related to each other. Intending to set examples and clarify the way in which the authors are understanding and handling data.

Qualitative approaches are well-established methods of analysis in areas such as Psychology and Sociology. Currently in Transportation Planning and Engineering, they are being increasingly used in road safety and public transportation service quality studies. Furthermore, since the reviews carried out by Grosvenor (2000) and Clifton and Handy (2003), many researches have also employed qualitative methods in travel behavior studies.

The main objective of this paper is to analyze the application of qualitative methods in travel behavior studies, published since 2001. We have not considered freight, road safety nor transportation service quality studies. We focus on behavioral studies, analysis of attitudes and perceptions, and the influence of the urban environment or social interactions on travel behavior.

\section{LITERATURE REVIEW}

In pursuing the aim of this work, we have carried out a literature review through mainly two databases, "Google Scholar" and "Web of Science"; the timespan was set from 2001 to 2016; and the key words used were in essence "qualitative", "transport*" or "travel". The research domains were defined excluding those not related with our theme study, as previously explained.

The following table (Table 1) summarizes the main features of the 42 papers found in the literature search. The authors' names, and publication year, travel modes involved in the study, and the main objective of the study are presented. Regarding methodology, the qualitative data collection method or methods used in each study are included; the number or participants; if the study has combined qualitative with quantitative techniques or not; and whether computerized qualitative data processing software has been used any to analyze data.

Table 1 also includes the analysis technique employed, if it is specified in the text. Finally, we analyzed if each paper details the procedure of the data collection, and the application of the data interpretation technique, including coding and data analysis (see Table 1). 
Table 1. Literature review qualitative travel behavior

\begin{tabular}{|c|c|c|c|c|c|c|c|c|c|}
\hline Authors & Year & Travel Mode & Objective & Data collection methods & Participants & $\begin{array}{l}\text { Method o } \\
\text { analysis }\end{array}$ & Software & Data Analysis Technique & $\begin{array}{c}\text { Methodological Description of } \\
\text { Qualitative Analysis }\end{array}$ \\
\hline $\begin{array}{l}\text { Handy, S.L., \& } \\
\text { Clifton, K.J. }\end{array}$ & 2001 & Car & $\begin{array}{l}\text { Possibility to reduce car } \\
\text { using by providing local } \\
\text { shopping opportunities }\end{array}$ & $\begin{array}{l}\text { Household travel survey } \\
\text { Focus groups }\end{array}$ & $\begin{array}{l}6 \text { Focus groups (unknown } \\
\text { number of participants) }\end{array}$ & Mixed & & Not specified & Not explained \\
\hline $\begin{array}{l}\text { Seedat, M., } \\
\text { MacKenzie, S., \& } \\
\text { Mohan, D. }\end{array}$ & 2006 & Pedestrians & $\begin{array}{l}\text { Pedestrian behaviors in } \\
\text { females students in Africa } \\
\text { and Asia }\end{array}$ & Interviews & 19 participants & \multicolumn{2}{|l|}{ Qualitative } & $\begin{array}{l}\text { Phenomenological approach } \\
\text { An integrated eight step data } \\
\text { collection and analytical } \\
\text { approach (Combining } \\
\text { different approaches) }\end{array}$ & $\begin{array}{l}\text { Data collection Coding and } \\
\text { analysis thoroughly explained }\end{array}$ \\
\hline $\begin{array}{l}\text { Beirao, G., \& } \\
\text { Sarsfield-Cabral, J.A. }\end{array}$ & 2007 & $\begin{array}{l}\text { Public } \\
\text { transport }\end{array}$ & $\begin{array}{l}\text { Perception of public } \\
\text { transport }\end{array}$ & In-depth interviews. & 24 in-depth interviews & Qualitative & NVivo 2.0. & Grounded theory Approach & $\begin{array}{l}\text { Data collection explained } \\
\text { Coding and analysis superficially } \\
\text { explained }\end{array}$ \\
\hline $\begin{array}{l}\text { Gardner,B., \& } \\
\text { Abraham, Ch. }\end{array}$ & 2007 & Car & Reasons to commute by car & Semi-structured interviews & $\begin{array}{l}19 \text { regular private car } \\
\text { commuters }\end{array}$ & \multicolumn{2}{|l|}{ Qualitative } & Grounded theory analysis & $\begin{array}{l}\text { Data collection explained } \\
\text { Coding and analysis superficially } \\
\text { explained }\end{array}$ \\
\hline Baslington, $\mathrm{H}$. & 2008 & $\begin{array}{l}\text { General } \\
\text { travel } \\
\text { behavior }\end{array}$ & Route choice & $\begin{array}{l}\text { Questionnaires } \\
\text { Travel diaries } \\
\text { Interviews }\end{array}$ & $\begin{array}{l}555 \text { questionnaires and } \\
\text { travel diaries } \\
22 \text { interviews and } 4 \text { key } \\
\text { person Interviews }\end{array}$ & Mixed & & $\begin{array}{l}\text { Case study } \\
\text { Content Analysis }\end{array}$ & $\begin{array}{l}\text { Data collection thoroughly } \\
\text { explained } \\
\text { Coding and analysis not } \\
\text { explained }\end{array}$ \\
\hline Farag, S., \& Lyons, G. & 2008 & $\begin{array}{l}\text { Public } \\
\text { transport }\end{array}$ & $\begin{array}{l}\text { Use of pre-trip public } \\
\text { transport information } \\
\text { services }\end{array}$ & $\begin{array}{l}\text { Face-to-face in-depth } \\
\text { interviews } \\
\text { focus groups (with two } \\
\text { travel scenarios and strategy } \\
\text { cards) }\end{array}$ & $\begin{array}{l}12 \text { face to face in-depth } \\
\text { interviews } \\
62 \text { people in six focus groups. }\end{array}$ & Qualitative & & Not specified & $\begin{array}{l}\text { Data collection thoroughly } \\
\text { explained } \\
\text { Coding and analysis not } \\
\text { explained }\end{array}$ \\
\hline $\begin{array}{l}\text { Lovehoy, K., \& } \\
\text { Handy, S }\end{array}$ & 2008 & Car & Car use and immigrants & Focus groups & $\begin{array}{l}102 \text { focus-group participants, } \\
\text { in five focus groups }\end{array}$ & Qualitative & & Not explained & Not explained \\
\hline $\begin{array}{l}\text { Hannes, E., Janssens, } \\
\text { D., \& Wets, G., }\end{array}$ & 2009 & $\begin{array}{l}\text { General } \\
\text { travel } \\
\text { behavior }\end{array}$ & Mental map travel behavior & $\begin{array}{l}\text { A qualitative travel survey } \\
\text { In-depth interviews }\end{array}$ & 20 respondents & Qualitative & ATLAS.ti. & $\begin{array}{l}\text { Descriptive, Explorative } \\
\text { analysis } \\
\text { Grounded Theory. } \\
\text { Cross-case analysis }\end{array}$ & $\begin{array}{l}\text { Data collection coding and } \\
\text { analysis thoroughly explained }\end{array}$ \\
\hline
\end{tabular}




\begin{tabular}{|c|c|c|c|c|c|c|c|c|c|}
\hline Authors & Year & Travel Mode & Objective & Data collection methods & Participants & $\begin{array}{l}\text { Method of } \\
\text { analysis }\end{array}$ & Software & Data Analysis Technique & $\begin{array}{c}\text { Methodological Description of } \\
\text { Qualitative Analysis }\end{array}$ \\
\hline $\begin{array}{l}\text { Papinski, D., Scott, } \\
\text { D.M., \& Doherte, S.T. }\end{array}$ & 2009 & Car & Route choice & $\begin{array}{l}\text { Audio recording, Diaries, } \\
\text { Route planning, Route } \\
\text { choice survey, Open ended } \\
\text { questions, Rank ordering,... } \\
\text { Follow-up questions }\end{array}$ & $\begin{array}{l}31 \text { individuals } \\
21 \text { vehicle based trips }\end{array}$ & Mixed & & Not specified & $\begin{array}{l}\text { Coding and analysis superficially } \\
\text { explained }\end{array}$ \\
\hline $\begin{array}{l}\text { Fleiter, J.J., Lennon, } \\
\text { A., \& Watson, B. }\end{array}$ & 2010 & Car & $\begin{array}{l}\text { Social influence on driving } \\
\text { speeds }\end{array}$ & $\begin{array}{l}\text { Focus groups } \\
\text { semi-structured interview } \\
\text { format using open-ended } \\
\text { questions }\end{array}$ & 67 Australian drivers & Qualitati & & Thematic analysis & $\begin{array}{l}\text { Data collection explained } \\
\text { Coding and analysis barely } \\
\text { explained }\end{array}$ \\
\hline Daley, M., \&Rissel, C. & 2011 & Bicycle & Perception of cycle & Focus groups & 70 participants. & Qualitat & NVivo7 & $\begin{array}{l}\text { Thematically analyzed. } \\
\text { Template analysis }\end{array}$ & $\begin{array}{l}\text { Data collection thoroughly } \\
\text { explained and coding explained }\end{array}$ \\
\hline Kopnina, $\mathrm{H}$. & 2011 & Car & $\begin{array}{l}\text { Children's attitudes toward } \\
\text { cars and environment }\end{array}$ & $\begin{array}{l}\text { Writing Assignments } \\
\text { interviews }\end{array}$ & $\begin{array}{l}69 \text { children completed the } \\
\text { written assignment } \\
9 \text { follow-up interviews }\end{array}$ & Qualitat & MAXQDA & $\begin{array}{l}\text { Case study } \\
\text { Content analysis }\end{array}$ & $\begin{array}{l}\text { Data collection and coding } \\
\text { explained }\end{array}$ \\
\hline $\begin{array}{l}\text { Lovehoy, K., \& } \\
\text { Handy, S }\end{array}$ & 2011 & Car & Car use and immigrants & Focus groups & $\begin{array}{l}102 \text { focus-group participants, } \\
\text { in five focus group }\end{array}$ & Qualitat & & Content for analysis & Not explained \\
\hline $\begin{array}{l}\text { Mote, J.E., \& } \\
\text { Whitstone, Y. }\end{array}$ & 2011 & Car & $\begin{array}{l}\text { Exploring slugging, } \\
\text { carpooling }\end{array}$ & $\begin{array}{l}\text { In-depth semi structured } \\
\text { interviews }\end{array}$ & $\begin{array}{l}12 \text { in-depth, semi structured } \\
\text { interviews }\end{array}$ & Qualitat & & Not specified & coding and analysis explained \\
\hline $\begin{array}{l}\text { Salomon, I., \& Singer } \\
\text { R. }\end{array}$ & 2011 & $\begin{array}{l}\text { General } \\
\text { travel } \\
\text { behavior }\end{array}$ & (Cartoons and transport) & $\begin{array}{l}\text { Various collections and } \\
\text { artists. (Including The New } \\
\text { Yorker, T. McCracken, John } \\
\text { Heine, Alex Hughes and } \\
\text { architect David Macaulay, } \\
\text { among others) }\end{array}$ & $\begin{array}{l}43 \text { cartoons about } \\
\text { transportation humor }\end{array}$ & Qualitat & & Inductive thematic analysis & $\begin{array}{l}\text { Data collection explained } \\
\text { Coding and analysis superficially } \\
\text { explained }\end{array}$ \\
\hline Schneider, R.J., & 2011 & $\begin{array}{l}\text { Bicycle and } \\
\text { pedestrians }\end{array}$ & $\begin{array}{l}\text { Walking and cycling for } \\
\text { routine travel }\end{array}$ & $\begin{array}{l}\text { Survey } \\
\text { Follow- up telephone } \\
\text { interviews }\end{array}$ & $\begin{array}{l}1,003 \text { survey respondents } \\
26 \text { follow-up interviews }\end{array}$ & Mixed & & Thematic analysis & $\begin{array}{l}\text { Coding and analysis not } \\
\text { explained }\end{array}$ \\
\hline
\end{tabular}




\begin{tabular}{|c|c|c|c|c|c|c|c|c|c|}
\hline Authors & Year & Travel Mode & Objective & Data collection methods & Participants & $\begin{array}{l}\text { Method of } \\
\text { analysis }\end{array}$ & Software & Data Analysis Technique & $\begin{array}{c}\text { Methodological Description of } \\
\text { Qualitative Analysis }\end{array}$ \\
\hline $\begin{array}{l}\text { Wilton, R.D., Páez, } \\
\text { A., \& Scott, D.M. }\end{array}$ & 2011 & $\begin{array}{l}\text { General } \\
\text { travel } \\
\text { behavior }\end{array}$ & $\begin{array}{l}\text { Social contact and } \\
\text { telecommuting }\end{array}$ & Semi-structured interviews. & 32 interviews & Qualitative & NUD_IST & $\begin{array}{l}\text { Inductive and deductive } \\
\text { analysis } \\
\text { Mixed method: grounded } \\
\text { theory and case study and } \\
\text { 'selective coding' approach }\end{array}$ & $\begin{array}{l}\text { Coding and analysis not } \\
\text { explained }\end{array}$ \\
\hline $\begin{array}{l}\text { Fishman, E., } \\
\text { Washington, S., \& } \\
\text { Haworth, N. }\end{array}$ & 2012 & Bicycle & $\begin{array}{l}\text { Perception of cycle and } \\
\text { bicycle share }\end{array}$ & Focus groups & 30 people 5 focus groups & Qualitative & & $\begin{array}{l}\text { Inductive analysis } \\
\text { Thematic analytic. (A process } \\
\text { similar to the first two stages } \\
\text { of Grounded Theory was } \\
\text { employed) }\end{array}$ & $\begin{array}{l}\text { Data collection coding and } \\
\text { analysis thoroughly explained }\end{array}$ \\
\hline $\begin{array}{l}\text { Graham-Rowe, E., } \\
\text { Gardner, B., } \\
\text { Abraham, C., } \\
\text { Skippon, S., } \\
\text { Dittmar, H., } \\
\text { Hutchins, R., \& } \\
\text { Stannard, J. }\end{array}$ & 2012 & Car & Perception of electric cars & $\begin{array}{l}\text { Semi-structured interview } \\
\text { open-ended questions }\end{array}$ & $\begin{array}{l}40 \text { UK non-commercial } \\
\text { drivers }\end{array}$ & Qualitative & & $\begin{array}{l}\text { Inductive analysis } \\
\text { Grounded theory analysis }\end{array}$ & $\begin{array}{l}\text { Data collection coding and } \\
\text { analysis thoroughly explained }\end{array}$ \\
\hline $\begin{array}{l}\text { Bartle, C., Avineri, E., } \\
\text { \& Chatterjee, K. }\end{array}$ & 2013 & Bicycle & Perception of cycle & $\begin{array}{l}\text { Observation of website } \\
\text { interactions (Cycology), } \\
\text { Questionnaires open } \\
\text { questions Semi-structured } \\
\text { In-depth interviews }\end{array}$ & $\begin{array}{l}23 \text { people hinge the Cycology } \\
\text { website } \\
21 \text { interviews }\end{array}$ & Qualitative & NVivo & $\begin{array}{l}\text { Case study } \\
\text { Holistic and Thematic analysis } \\
\text { (Horizontal and vertical } \\
\text { respectively) }\end{array}$ & $\begin{array}{l}\text { Collection coding and analysis } \\
\text { explained }\end{array}$ \\
\hline $\begin{array}{l}\text { Chatman, D.G., \& } \\
\text { Klein, N.J. }\end{array}$ & 2013 & car & Car use and immigrants & Focus groups & $\begin{array}{l}55 \text { participants in six focus } \\
\text { group }\end{array}$ & Qualitative & $\begin{array}{l}\text { Yes but not } \\
\text { specified }\end{array}$ & $\begin{array}{l}\text { Inductive and deductive codes } \\
\text { Iterative process employing }\end{array}$ & $\begin{array}{l}\text { Data collection explained } \\
\text { Coding and analysis superficially } \\
\text { explained }\end{array}$ \\
\hline $\begin{array}{l}\text { Lo, S.H., van } \\
\text { Breukelen, G.J.P., } \\
\text { Peters, G.J., \& Kok, } \\
\text { G. }\end{array}$ & 2013 & $\begin{array}{l}\text { Various } \\
\text { modes }\end{array}$ & $\begin{array}{l}\text { Determinants of work- } \\
\text { related travel behavior }\end{array}$ & $\begin{array}{l}\text { Semi-structured Interviews } \\
\text { Focus groups }\end{array}$ & $\begin{array}{l}18 \text { interviews to Key } \\
\text { informants } \\
33 \text { interviews to Employees } \\
6 \text { Focus Group with } 31 \\
\text { participants }\end{array}$ & Qualitative & NVivo 8 & thematic analysis & $\begin{array}{l}\text { Data collection coding and } \\
\text { analysis thoroughly explained }\end{array}$ \\
\hline
\end{tabular}




\begin{tabular}{|c|c|c|c|c|c|c|c|c|c|}
\hline Authors & Year & Travel Mode & Objective & Data collection methods & Participants & $\begin{array}{l}\text { Method } \\
\text { analysis }\end{array}$ & Software & Data Analysis Technique & $\begin{array}{l}\text { Methodological Description of } \\
\text { Qualitative Analysis }\end{array}$ \\
\hline $\begin{array}{l}\text { Pooley, C.G., } \\
\text { Horton,D., } \\
\text { Scheldeman, G., } \\
\text { Mullen, C., Jones, T., } \\
\text { Tight, M., Jopson, A., } \\
\text { \& Chisholm, A. }\end{array}$ & 2013 & $\begin{array}{l}\text { Bicycle and } \\
\text { pedestrians }\end{array}$ & $\begin{array}{l}\text { Travel decision and walking } \\
\text { and cycling }\end{array}$ & $\begin{array}{l}\text { Postal questionnaire survey } \\
\text { Spatial analysis of the } \\
\text { connectivity of all usable } \\
\text { Routes interviews } \\
\text { (household and while } \\
\text { travelling) } \\
\text { Ethnographies }\end{array}$ & $\begin{array}{l}\text { Postal questionnaire survey } \\
\text { sent to } 15,000 \\
80 \text { interviews with } \\
\text { households individuals } \\
20 \text { household ethnographies }\end{array}$ & Qualitativ & ATLAS.ti. & $\begin{array}{l}\text { Case study } \\
\text { Ethnography study }\end{array}$ & $\begin{array}{l}\text { Coding and analysis not } \\
\text { explained }\end{array}$ \\
\hline Schneider, R.J. & 2013 & $\begin{array}{l}\text { Bicycle and } \\
\text { pedestrians }\end{array}$ & $\begin{array}{l}\text { Incrementing pedestrian } \\
\text { and bicycle transportation }\end{array}$ & $\begin{array}{l}\text { Survey } \\
\text { In-depth interview responses }\end{array}$ & $\begin{array}{l}172 \text { survey participants } \\
\text { s } 26 \text { people interviewed }\end{array}$ & Qualitativ & & Not specified & $\begin{array}{l}\text { Coding and analysis not } \\
\text { explained }\end{array}$ \\
\hline $\begin{array}{l}\text { Delbosc A,. \& Currie, } \\
\text { G. }\end{array}$ & 2014 & Car & $\begin{array}{l}\text { Perception of the youth } \\
\text { about cars and license } \\
\text { acquisition }\end{array}$ & $\begin{array}{l}\text { Asynchronous discussion } \\
\text { forum }\end{array}$ & 33 people in 3 focus group & Qualitativ & & Thematic Analysis. & $\begin{array}{l}\text { Data collection thoroughly } \\
\text { explained } \\
\text { Coding methodology not } \\
\text { explained }\end{array}$ \\
\hline $\begin{array}{l}\text { Krishen, A.S., } \\
\text { Raschke, R.L., } \\
\text { Kachroo,P., Mejza, } \\
\text { M., \& Khan, A. }\end{array}$ & 2014 & Car & $\begin{array}{l}\text { Analyzing public } \\
\text { commentaries toward } \\
\text { potential Vehicle Miles } \\
\text { Traveled (VMT) }\end{array}$ & $\begin{array}{l}\text { Transcription of public } \\
\text { comments } \\
\text { Mails and comments } \\
\text { Comment postings from } \\
\text { newspapers }\end{array}$ & $\begin{array}{l}14 \text { Public meeting comments } \\
182 \text { NDOT VMT study } \\
\text { Internet communications } \\
\text { comments and emails } \\
97 \text { Newspaper article } \\
\text { comments } \\
\text { From } 293 \text { different } \\
\text { individuals }\end{array}$ & Qualitativ & Leximancer & $\begin{array}{l}\text { Inductive qualitative-analysis } \\
\text { technique } \\
\text { Content analysis and media } \\
\text { mode analysis }\end{array}$ & $\begin{array}{l}\text { Data collection, coding and } \\
\text { analysis explained }\end{array}$ \\
\hline $\begin{array}{l}\text { Miralles-Guasch, C., } \\
\text { Martínez, M., \& } \\
\text { Sardà, O. }\end{array}$ & 2014 & Car & Reasons for car commuting & In-depth interviews & 34 interviews & Mixed & & Grounded theory & Not explained \\
\hline $\begin{array}{l}\text { Sherwin, H., } \\
\text { Chatterjeem K., \& } \\
\text { Jain, J. }\end{array}$ & 2014 & Bicycle & Perception of cycle & Interviews & 61 Interviews & Qualitativ & NVivo & $\begin{array}{l}\text { Thematic analysis. } \\
\text { Systematic approach }\end{array}$ & $\begin{array}{l}\text { Coding and analysis superficially } \\
\text { explained }\end{array}$ \\
\hline $\begin{array}{l}\text { Simons, D., Clarys, P., } \\
\text { Bourdeaudhuij, l., } \\
\text { Geus, B., } \\
\text { Vandelanotte, C., \& } \\
\text { Deforche, B. }\end{array}$ & 2014 & $\begin{array}{l}\text { Various } \\
\text { modes }\end{array}$ & $\begin{array}{l}\text { Factors influencing } \\
\text { transport mode }\end{array}$ & Focus groups & 36 people in 6 focus groups & Qualitativ & NVivo 9 & $\begin{array}{l}\text { Grounded theory was used to } \\
\text { derive categories and } \\
\text { subcategories }\end{array}$ & $\begin{array}{l}\text { Coding and analysis not } \\
\text { explained }\end{array}$ \\
\hline
\end{tabular}




\begin{tabular}{|c|c|c|c|c|c|c|c|c|c|}
\hline Authors & Year & Travel Mode & Objective & Data collection methods & Participants & \multicolumn{2}{|l|}{$\begin{array}{l}\text { Method of } \\
\text { analysis }\end{array}$} & Data Analysis Technique & $\begin{array}{c}\text { Methodological Description of } \\
\text { Qualitative Analysis }\end{array}$ \\
\hline Skippon, S.M. & 2014 & Car & Vehicle performance & $\begin{array}{l}\text { Initial discussion of vehicle } \\
\text { performance (by triads) } \\
\text { using various stimulus } \\
\text { materials } \\
\text { Repertory grid completion } \\
\text { exercise }\end{array}$ & 48 participants & Mixed & & $\begin{array}{l}\text { Inductive phenomenological } \\
\text { analysis } \\
\text { Thematic analysis at a } \\
\text { semantic level } \\
\text { Cohen's Kappa test" }\end{array}$ & $\begin{array}{l}\text { Data collection, Coding and } \\
\text { analysis thoroughly explained }\end{array}$ \\
\hline $\begin{array}{l}\text { Thomas, G.O., } \\
\text { Walker, I., \& } \\
\text { Musselwhite, C. }\end{array}$ & 2014 & $\begin{array}{l}\text { Various } \\
\text { modes }\end{array}$ & $\begin{array}{l}\text { Different modes for work } \\
\text { commuting }\end{array}$ & Focus groups & $\begin{array}{l}27 \text { participants in } 6 \text { focus } \\
\text { groups. }\end{array}$ & Qualitative & & Grounded Theory & Coding and analysis explained \\
\hline $\begin{array}{l}\text { Aarhaug, J., \& } \\
\text { Elvebakk, B }\end{array}$ & 2015 & $\begin{array}{l}\text { Public } \\
\text { transport }\end{array}$ & $\begin{array}{l}\text { Accessibility of public } \\
\text { transport }\end{array}$ & $\begin{array}{l}\text { Surveys } \\
\text { An observer accompanying } \\
\text { Interviews }\end{array}$ & $\begin{array}{l}1.912 \text { surveys were } \\
\text { distributed in the before } \\
\text { study and } 1.361 \text { in the after } \\
\text { study. } \\
17 \text { case studies (before study) } \\
\& 6 \text { (after study) }\end{array}$ & Mixed & & Not specified & $\begin{array}{l}\text { Data collection explained } \\
\text { Coding and analysis not } \\
\text { explained }\end{array}$ \\
\hline $\begin{array}{l}\text { Aldred, R., \& } \\
\text { Woodcoc,k J. }\end{array}$ & 2015 & Bicycle & Perception of cycle & Interviews & 300 Interviews & Qualitative & NVivo & Thematic analysis & $\begin{array}{l}\text { Data collection, coding and } \\
\text { analysis explained }\end{array}$ \\
\hline $\begin{array}{l}\text { Grisolía, J.M., López, } \\
\text { F., \& Ortúza, J.D. }\end{array}$ & 2015 & Car & $\begin{array}{l}\text { Factors to accept congestion } \\
\text { charging }\end{array}$ & $\begin{array}{l}\text { Focus groups } \\
\text { Questionnaires Likert scales } \\
\text { Stated choice (SC) }\end{array}$ & $\begin{array}{l}81 \text { participants in } 10 \text { focus } \\
\text { groups. } \\
206 \text { respondents Stated } \\
\text { Choice experiment }\end{array}$ & Mixed & & Content analysis & $\begin{array}{l}\text { Data collection explained. } \\
\text { Coding and analysis barely } \\
\text { explained }\end{array}$ \\
\hline $\begin{array}{l}\text { Haupt, J., van Nes, } \\
\text { N., \& Risser, R. }\end{array}$ & 2015 & Car & Route choice & $\begin{array}{l}\text { Video recording observation } \\
\text { analysis }\end{array}$ & 20 participants & Mixed & $\begin{array}{l}\text { Cameras } \\
\text { and not } \\
\text { specified } \\
\text { data } \\
\text { reduction } \\
\text { software } \\
\end{array}$ & Video observation analysis & $\begin{array}{l}\text { Data collection thoroughly } \\
\text { explained } \\
\text { Coding and analysis superficially } \\
\text { explained }\end{array}$ \\
\hline $\begin{array}{l}\text { Kaparias, I., Bell, } \\
\text { M.G.H., Biagioli, T., } \\
\text { Bellezza, L., \& } \\
\text { Mount, B. }\end{array}$ & 2015 & Car & $\begin{array}{l}\text { Pedestrians and drivers } \\
\text { behavior }\end{array}$ & $\begin{array}{l}\text { Video observation and } \\
\text { coding. } \\
\text { This has also been } \\
\text { complemented by vehicle } \\
\text { traffic and pedestrian } \\
\text { crossing counts. }\end{array}$ & $\begin{array}{l}\text { Video observation of vehicle- } \\
\text { pedestrian interaction from } \\
2008 \text { to } 2011 . \\
\text { Exhibition Road is an } 800 \mathrm{~m} \\
\text { long road located in West } \\
\text { London and is home to a } \\
\text { number of London's most } \\
\text { popular museums (Natural } \\
\text { History, Science, V\&A). }\end{array}$ & Mixed & $\begin{array}{l}\text { Video } \\
\text { observation } \\
\text { software } \\
\text { not } \\
\text { specified }\end{array}$ & $\begin{array}{l}\text { Case study } \\
\text { Video observation, } \\
\text { behavioral analysis method } \\
\text { introduced consists of three } \\
\text { steps }\end{array}$ & $\begin{array}{l}\text { Data collection Thoroughly } \\
\text { explained } \\
\text { Coding superficially explained }\end{array}$ \\
\hline
\end{tabular}


Nielsen, J.R.;
Hovmøller, H., Blyth, 2015 Car

P.L., \& Sovacool, B.K.
Semi-structured research

interviews

Focus groups
Roughly 50 people attended

Inductive analysis

Grounded theory ata collection explained

a qualitative version of factor Coding and analysis not

analysis

explained

\begin{tabular}{|c|c|c|c|c|c|c|c|c|c|}
\hline Nostilasari, D. & 2015 & $\begin{array}{l}\text { Various } \\
\text { modes }\end{array}$ & $\begin{array}{l}\text { Transportation needs of } \\
\text { various population groups }\end{array}$ & $\begin{array}{l}\text { One-week travel diary (GPS } \\
\text { recorder, travel form, and } \\
\text { images) } \\
\text { Semi-structured interviews }\end{array}$ & 15 participants & $\begin{array}{l}\text { Qualitative } \\
\text { (and GPS } \\
\text { data) }\end{array}$ & & Case study & $\begin{array}{l}\text { Data collection explained } \\
\text { Coding and analysis superficially } \\
\text { explained }\end{array}$ \\
\hline $\begin{array}{l}\text { Spotswood, F., } \\
\text { Chatterton, T., Tapp, } \\
\text { A., \& Williams, D. }\end{array}$ & 2015 & Bicycle & Cycling as a social issue & $\begin{array}{l}\text { Study 1: online survey } \\
\text { (quantitative) } \\
\text { Study 2: depth interviews } \\
\text { and focus groups included a } \\
\text { 'psycho-drawing' exercise. }\end{array}$ & $\begin{array}{l}\text { Study 1: } 3885 \text { online survey } \\
\text { Study 2: } 10 \text { depth interviews } \\
\text { and } \quad 60 \text { participants in } 9 \\
\text { focus groups }\end{array}$ & $\begin{array}{l}\text { Mixed } \\
\text { Quantitative } \\
\text { and } \\
\text { Qualitative }\end{array}$ & Vvivo & Thematic analysis & $\begin{array}{l}\text { Data collection thoroughly } \\
\text { explained } \\
\text { Coding and analysis not } \\
\text { explained }\end{array}$ \\
\hline $\begin{array}{l}\text { Ferrer, S., Ruiz, T., \& } \\
\text { Mars L. }\end{array}$ & 2015 & Pedestrians & & $\begin{array}{l}\text { Focus groups (With } \\
\text { photographs showed to } \\
\text { focus groups) }\end{array}$ & 23 participants & Qualitative & NVivo 10 & $\begin{array}{l}\text { A thematic analysis of the } \\
\text { data }\end{array}$ & $\begin{array}{l}\text { Data collection, Coding and } \\
\text { analysis explained }\end{array}$ \\
\hline $\begin{array}{l}\text { Cass, N., \& } \\
\text { Faulconbridge, J. }\end{array}$ & 2016 & Car & $\begin{array}{l}\text { Shifting from car to other } \\
\text { more friendly transport } \\
\text { mode }\end{array}$ & Semi-structured interviews & $\begin{array}{l}101 \text { semi-structured } \\
\text { interviews }\end{array}$ & Qualitative & NVivo & $\begin{array}{l}\text { Grounded Theory Approach } \\
\text { Analytic approach combining } \\
\text { inductive and deductive } \\
\text { techniques }\end{array}$ & $\begin{array}{l}\text { Data collection explained } \\
\text { Coding and analysis not } \\
\text { explained }\end{array}$ \\
\hline $\begin{array}{l}\text { Karndacharuk, A., } \\
\text { Wilson, D.J., \& Dunn, } \\
\text { R.C.M. }\end{array}$ & 2016 & $\begin{array}{l}\text { Various } \\
\text { modes } \\
\text { including } \\
\text { pedestrians }\end{array}$ & $\begin{array}{l}\text { Shared streets, points of } \\
\text { view pedestrian and } \\
\text { vehicles }\end{array}$ & $\begin{array}{l}\text { On-street perception surveys } \\
\text { Expert interview surveys }\end{array}$ & $\begin{array}{l}360 \text { responses on-street } \\
\text { perception surveys } \\
40 \text { responses of a control site } \\
\text { that remained as a traditional } \\
\text { street survey } \\
15 \text { professional semi- } \\
\text { structured expert interviews }\end{array}$ & $\begin{array}{l}\text { Mixed } \\
\text { Quantitative } \\
\text { and } \\
\text { Qualitative }\end{array}$ & & Not specified & $\begin{array}{l}\text { Data collection explained } \\
\text { Coding and analysis not } \\
\text { explained }\end{array}$ \\
\hline
\end{tabular}




\section{ANALYSIS}

In our review, we have detected an increasing interest in applying qualitative methodology in recent years. The number of qualitative articles published since 2010 is higher than in the previous years.

Regarding the travel modes involved in the study, a higher number of articles referring to topics related to cars (19 up to 42 studies); followed by articles that focuses on pedestrians $(n=7)$, bicycle issues $(n=6)$, various modes of transport $(n=5)$, general travel behavior $(n$ $=4)$ and lastly public transport $(n=3)$. In recent years, there are more studies focuses on bicycles and pedestrians, or evaluation of different transport modes.

Among the major topics found in our literature review we can see the concern on reducing gasoline or diesel cars use for the benefit of other more sustainable travel modes like electric cars, cycling and walking. Other topics of interest are route choice and travel behavior controlling for demographics (age, immigrants).

About data collection methods, the interview is the most commonly used method. In-depth interviews have been used in nearly half of the articles reviewed, either face to face or over the phone. The following method is conducting focus groups, in which participants are encouraged to present and discuss their own points of view. They usually have a semistructured discussion guide and a specific timing. In those focus groups, there are present two researchers, one leading or facilitating the group and the other controlling, supporting, or taking notes.

A point we would like to highlight is the use of other techniques to help focus groups. Researchers knows the benefits of the use of certain procedures or resources in order to get the best possible performance out of the focus group. For example, we have found techniques like psycho-drawing, using verbatim notes from other people, playing video clips of driving situations, audiovisual recordings, showing photographs or travel scenarios.

The third method used by researchers is making surveys or questionnaires with open-ended questions. Similarly, this method allows building an integrated analysis and obtaining at the same time qualitative and quantitative data. In other cases it allows getting responses to very specific questions.

We have found other methods to collect data, like, diaries, ethnographies, grid completion exercises by triads, video recording and even individual cartoons. It deserves also special mention those methods related to data extracted from online social media.

In addition, nearly half the studies reviewed use several methods simultaneously to collect information, such semi structured questionnaires with open-ended questions and focus 
groups; or diaries followed by focus groups or individual interviews.

Different methods for recruiting participants are used. For example, purposive sampling, which consists on selecting individuals or cases that represent the population average, or extreme (deviant) or disconfirming cases (negative) (Devers \& Frankel, 2000). Other method is snowball sampling, which is a technique consisting in the recruitment of subjects by other subjects already in the study because they are relatives, colleagues or acquaintances (Goodman, 1961). Convenience sample, in which the subjects are selected because of their convenient accessibility and proximity to the researcher (Farrokhi \& MahmoudiHamidabad, 2012).

Only one third of all papers reviewed combined qualitative and quantitative methodologies of analysis. Some of them use qualitative prior to quantitative analysis (i.e. video observation and posterior ratio analysis). Others use qualitative after the quantitative is undertaken (i.e. first they collect data from a survey and them they make focus group or follow-up interviews). In addition, others use qualitative to complement quantitative findings (i.e. interviewing key persons, or using case studies to deepen the results).

18 up to 42 studies declare using some software to categorize data. 11 of them use QSR International's NVivo qualitative data analysis Software. Two of them use Atlas.ti. And others used MAXQDA and LEXIMANCE, or video observation software.

Regarding to the data analysis technique used in the study, nine of them refers to us Inductive Analysis and three Deductive Analysis, although the latter also used inductive analysis. The most commonly used technique is Thematic Analysis (13 articles). This technique examines and search for patterns or themes within the data (Braun \& Clarke, 2006). The following more used technique is Grounded Theory (Glasser \& Strauss, 1967); where the data is clustered in the following order into codes, concepts, categories finally a theory. Seven of the articles use Case Studies where a person or a group is studied over time. Four articles use Content Analysis, which consists on selecting the unit of analysis, creating categories, and establishing themes (Cho \& Lee, 2014). Two of the studies perform a video observation. Finally, Ethnographic Descriptive analysis and Template Analysis are also utilized. Noteworthy, there are seven articles that do not specify explicitly the data analysis technique that they are using.

In order to increase consistency, clarity and congruence, the methodological qualitative analysis needs to be carefully described in any paper. In most of the papers reviewed, the data collection methods is explained at length, in 14 cases thoroughly explained and in 16 cases there is a properly explanation. However, there are cases in which they do not provide any detail about how they carried out the interviews or focus groups, what kind of questions they used, how was the timing structured, and a number of other related issues.

In just over a third of the articles $(n=14)$ the coding and the analysis process is explained $(n$ 
$=7$ ) or thoroughly explained ( $n=7)$. In 9 articles it is superficially explained and in two there are hardly any information. But more remarkable is that in 15 of papers reviewed the data analysis process is not mentioned at all. Sometimes, a schematic explanation of how they arrived to the results is given. But it is not enough to understand the process followed.

\section{CONCLUSIONS}

We have noticed a greater increase of qualitative studies in the last years. The option to choose qualitative methods to perform travel behavior studies is becoming more present for researchers in Transport Planning and Engineering field.

The travel mode more broadly studied is car covering issues like car sharing, age and immigrants issues, shifting to more nature-friendly transportation modes, commuting or social influence on driving. However in the last years it seems to be an increasing interest in other travel modes like bicycles. In particular, the perceptions that cyclers on the one hand and drivers or pedestrians on the other have about people who uses bicycles not only for leisure but also for physical activity or medium-short displacements. There has also been an increase of studies interested on the experience of pedestrians either to learn what factors make their way more enjoyable or to analyze relationships between pedestrians and other modes of transport.

Interviews and focus groups are the most commonly methods to collect qualitative data. Many of the articles even include a detailed analysis of the participant recruitment, scripts of the questions asked, the timings for each question, or descriptions on the formation and development of focus groups.

Most of the papers reviewed expose that more than one person has studied the data and different tasks to avoid bias have been performed. However, that level of detail disappears when it involves exposing the method used or actions taken to elaborate categories or themes. The revised articles often jump into results without further explanation. This makes sometimes difficult to follow and understand the results in their full extent. As explained earlier, qualitative studies must have explicit how data analysis techniques have been applied. Otherwise, a potential replication of the research would be difficult.

It is observed an increasing tendency in relation to the use of software to support qualitative coding and analysis allowing handling larger amounts of information. It also allows reviewing the coding and categories at any time. The use of software can be very useful as a triangulation tool.

We are aware that this is not a comprehensive review of the existing scientific data. However, we consider that we have found and adequate quantity of studies to represent the current state of the art of qualitative method application on travel behavior studies. 


\section{REFERENCES}

Aarhaug, J. \& Elvebakk, B. (2015). The impact of universally accessible public transport-a before and after study. Transport Policy, 44, 143-150.

Aldred, R. \& Woodcoc,k J. (2015). Reframing safety: An analysis of perceptions of cycle safety clothing. Transport Policy, 42, 103-112.

Amezcua, M., Gálvez Toro, A. (2002). Los modos de análisis en investigación cualitativa en salud:perspectiva crítica y reflexiones en voz alta. Revista Española de Salud Publica, 76(5), 423-436.

Bartle, C., Avineri, E. \& Chatterjee, K. (2013). Online information-sharing: A qualitative analysis of community, trustand social influence amongst commuter cyclists in the UK. Transportation Research part F, 16, 60-72.

Baslington, H. (2008). School Travel Plans: Overcoming Barriers to Implementation. Transport Reviews, 28, 2, 239-258.

Beirao, G., Sarsfield-Cabral, J.A. (2007). Understanding attitudes towards public transport and private car: a qualitative study. Transport Policy, 14, 478-489.

Braun, V., \& Clarke, V. (2006). Using thematic analysis in psychology. Qualitative Research in Psychology 3 (2): 83

Cass, N. \& Faulconbridge, J. (2016). Commuting practices: New insights into modal shift from theories of social practice. Transport Policy, 45, 1-14.

Chatman, D. G. \& Klein, N.J. (2013). Why do immigrants drive less? Confirmations, complications, and new hypotheses from a qualitative study in New Jersey, USA. Transport Policy, 30, 336-344.

Charmaz, K. (1996). Grounded theory. In J.A. Smith, R. Harré, \& L. Van Langenhove (Eds.). Rethinking Methods in Psychology (pp. 27-49). London Sage Publications.

Cho, J. Y., \& Lee, E. H. (2014). Reducing confusion about grounded theory and qualitative content analysis: Similarities and differences. The Qualitative Report, 19(32), 1-21.

Clifton, K., \& Handy, S. (2003). Qualitative methods in travel behaviour research. In: Transport Survey Quality and Innovation, Proceedings of an International Conference on 
Transport Survey Quality and Innovation, Kruger Park, South Africa, 283-302.

Daley, M. \& Rissel, C. (2011) Perspectives and images of cycling as a barrier or facilitator of cycling. Transport Policy, 18, 211-216.

Delbosc A. \& Currie, G. (2014). Using discussion forums to explore attitudes toward cars and licensing among young Australians. Transport Policy, 31, 27-34.

Devers, K.J., \& Frankel, R.M. (2000). Study design in qualitative research—2: sampling and data collection strategies. Education for Health, 13, 263-271.

Elliott, R., \& Timulak, L. (2005). Descriptive and interpretive approaches to qualitative research. A handbook of research methods for clinical and health psychology, 147-159.

Farag, S. \& Lyons, G. (2008). What affects pre-trip public transport information use? Empirical results of a qualitative study. $87^{\text {th }}$ Annual Meeting of Transportation Research Board, Washington DC.

Farrokhi, F., \& Mahmoudi-Hamidabad, A. (2012). Rethinking Convenience Sampling: Defining Quality Criteria. Theory and Practice in Language Studies, 2 (4), 784-792.

Ferrer, S., Ruiz, T., \& Mars L. (2015). A qualitative study on the role of the built environment for short walking trips. Transportation Research Part F, 33, 141-160.

Fishman, E., Washington, S. \& Haworth, N. (2012). Barriers and facilitators to public bicycle scheme use: A qualitative approach. Transportation Research Part F, 15, 686-698.

Fleiter, J.J., Lennon, A. \& Watson, B. (2010). How do other people influence your driving speed? Exploring the 'who' and the 'how' of social influences on speeding. Transportation Research Part F, 13, 49-62.

Flick, U. (2014). An Introduction to Qualitative Research. Sage Publishing,

Gardner,B. \& Abraham, Ch. (2007). What drives car use? A grounded theory analysis of commuters' reasons for driving. Transportation Research Part F, 10, 187-200.

Glaser, B.G., \& Strauss, A.L. (1967) The discovery of grounded theory: strategies for qualitative research. Chicago.: Aldine.

Goodman, L.A. (1961). "Snowball sampling". Annals of Mathematical Statistics 32 (1): $148-170$

Graham-Rowe, E., Gardner, B., Abraham Ch, Skippon, S., Dittmar, H., Hutchins, R. \& Stannard, J. (2012). Mainstream consumers driving plug-in battery-electric and plug-in hybrid electric cars: A qualitative analysis of responses and evaluations. Transportation 
Research Part A, 46, 140-153.

Grisolía, J.M., López, F. \& Ortúzar, J.D. (2015). Increasing the acceptability of a congestion charging scheme. Transport Policy, 39, 37-47.

Grosvenor, T. (2000), "Qualitative Research in the Transport Sector. Resource paper for the Workshop on Qualitative/Quantitative Methods," Proceedings of an International Conference on Transport Survey Quality and Innovation. May 24-30, 1997 (Grainau, Germany), Transportation Research E-Circular, Number E-C008, August.

Handy, S.L. \& Clifton, K.J. (2001). Local shopping as a strategy for reducing automobile travel. Transportation, 28, 317-346.

Hannes, E., Janssens, D. and Wets, G. (2009). 'Does Space Matter? Travel Mode Scripts in Daily Activity Travel'. Environment and Behavior, 41, 1, 75-100.

Haupt, J., van Nes, N. \& Risser, R. (2015). Look where you have to go! A field study comparing looking behaviour at urban intersections using a navigation system or a printed route instruction. Transportation Research Part F, 34, 122-140.

Hesse-Biber, S. (2010). Qualitative approaches to mixed methods practice. Qualitative Inquiry, 16, 455-468.

Hjorthol, R. (2001). Gendered aspects of time related to everyday journeys. Acta Sociológica.

Lo, S.H., van Breukelen, G.J.P. , Peters, G.J.\& Kok, G. (2013). Proenvironmental travel behavior among office workers: A qualitative study of individual and organizational determinants. Transportation Research Part A-Policy and Practice, 56, 11-22.

Madill, A., Jordan, A., \& Shirley, C., (2000). Objectivity and reliability in qualitative analysis: realist, contextualist and radical constructionist epistemologies. British Journal of Psychology 91, 1-20.

Kaparias, I., Bell, M.G.H., Biagioli, T., Bellezza, L. \& Mount, B. (2015). Behavioural analysis of interactions between pedestrians and vehicles in street designs with elements of shared space. Transportation Research Part F, 30, 115-127.

Karndacharuk, A.,Wilson, D.J., \&Dunn, R.C.M. (2016). Qualitative evaluation study of urban shared spaces in New Zealand. Transportation Research Part D, 42 119-134.

Kopnina, H. (2011). Kids and cars: Environmental attitudes in children. Transport Policy, $18,573-578$.

Krishen, A.S., Raschke,, R.L., Kachroo,P., Mejza, M., \& Khan, A. (2014). Interpretation of Public Feedback to Transportation Policy: A Qualitative Perspective. Transportation Journal, 53, 1, 26-43. 
Lovehoy, K., \& Handy, S. (2008). A case for measuring individuals' access to privatevehicle travel as a matter of degrees: lessons from focus groups with Mexican immigrants in California. Transportation, 35, 601-612.

Lovehoy, K., \& Handy, S. (2011). Social networks as a source of private-vehicle transportation: The practice of getting rides and borrowing vehicles among Mexican immigrants in California. Transportation Research Part A, 45, 248-257.

Madill, A., \& Gough, B. (2008). Qualitative research and its place in psychological science. Psychological Methods, 13, 254-271.

Miralles-Guasch, C., Montserrat Martínez Melo M. y Marquet Sardà O. (2014). On user perception of private transport in Barcelona Metropolitan area: an experience in an academic suburban space. Journal of Transport Geography, 36, 24-31.

Mote, J.E. \& Whitstone, Y. (2011). The social context of informal commuting: Slugs, strangers and structuration. Transportation Research Part A, 45, 258-268.

Nielsen, J.R.; Hovmøller, H., Blyth, P.L., \& Sovacool, B.K. (2015). Of "white crows”' and "cash savers:" A qualitative study of travel behavior and perceptions of ridesharing in Denmark. Transportation Research Part A, 78, 113-123.

Niglas, K. (2000). Combining quantitative and qualitative approaches. Proc. European Conference on Educational Research, Edinburgh, 20-23 September 2000.

Nostilasari, D. (2015). Representations of everyday travel experiences: Case study of the Dallas-Fort Worth Metropolitan Area. Transport Policy, 44, 96-107.

Papinski, D., Scott, D.M., \& Doherte, S.T. (2009). Exploring the route choice decisionmaking process: A comparison of planned and observed routes obtained using person-based GPS. Transportation Research Part F, 12, 347-358.

Patton, M.Q. (2005). Qualitative Research. Wiley.

Pooley, C.G., Horton,D., Scheldeman, G., Mullen, C., Jones, T., Tight, M., Jopson, A, \& Chisholm, A. (2013). Policies for promoting walking and cycling in England: A view from the street. Transport Policy 27, 66-72.

Salomon, I., \& Singer, R. (2011). Why did the chicken cross the road, and what's funny about it? The role of transportation cartoons in social experiences. Transport Policy, 18, 112.

Schneider, R.J. (2013). Theory of routine mode choice decisions: An operational framework of increase sustainable transportation. Transport Policy, 25, 128-137.

Schneider, R.J. (2011). Understanding Sustainable Transportation Choices: Shifting Routine Automobile Travel to Walking and Bicycling. Available online: /http://www.uctc.net/ research/UCTC-DISS-2011-01.pdfS. 
Seedat, M., MacKenzie, S., \& Mohan, D. (2006). The phenomenology of being a female pedestrian in an African and an Asian city: A qualitative investigation. Transportation Research Part F, 9, 139-153.

Sherwin, H., Chatterjeem K., \& Jain, J. (2014). An exploration of the importance of social influence in the decision to start bicycling in England. Transportation Research Part A, 68, $32-45$.

Simons, D., Clarys, P., Bourdeaudhuij, I., Geus, B., Vandelanotte, C., \& Deforche, B. (2014). Why do young adults choose different transport modes? A focu group study. Transport Policy, 36, 151-159.

Skippon, S.M. (2014). How consumer drivers construe vehicle performance: Implications for electric vehicles. Transportation Research Part F, 23, 15-31.

Smith, C.P. (2000). Content analysis and narrative analysis. In H.T. Reis \& C.M. Judd (eds), Handbook of research methods in social and personality psychology. New York: Cambridge University Press.

Spotswood, F., Chatterton, T., Tapp, A., \& Williams, D. (2015). Analysing cycling as a social practice: An empirical grounding for behaviour change. Transportation Research Part $F, 29,22-33$.

Thomas, G.O., Walker, I., \& Musselwhite, Ch. (2014). Grounded Theory analysis of commuters discussing a workplace carbon-reduction target: Autonomy, satisfaction, and willingness to change behaviour in drivers, pedestrians, bicyclists, motorcyclists, and bus users. Transportation Research Part F, 26, 72-81.

Wilton, R.D., Páez, A., \& Scott, D.M. (2011). Why do you care what other people think? A qualitative investigation of social influence and telecommuting. Transportation Research Part A, 45, 269-282.

Znaniecki, F. (1934). The method of sociology, New York, Farrar and Rinehart. 